\title{
Diecinueve años de investigación sobre el patrimonio paleobiológico de la Prehistoria Reciente andaluza
}

\author{
Esteban García-Viñas | Dpto. de Sistemas Físicos, Químicos y Naturales. U. Pablo \\ de Olavide \\ Aurora Ocaña | Paleobióloga, profesional libre \\ Miguel Gamero | Paeleobiólogo, profesional libre \\ Eloísa Bernáldez | Laboratorio de Paleontología y Paleobiología. IAPH; Dpto. de \\ Sistemas Físicos, Químicos y Naturales. U. Pablo de Olavide
}

URL de la contribución <www.iaph.es/revistaph/index.php/revistaph/article/view/3505>

\section{RESUMEN}

La paleobiología es una disciplina que estudia el registro fósil de animales y vegetales con fines biológicos, evolutivos y paleoecológicos. No obstante, cuando el material orgánico procede de yacimientos arqueológicos se utiliza para investigar las costumbres tróficas y basureras de los humanos a lo largo de la historia, además de para interpretar antiguos ecosistemas o el modo en que las sociedades pasadas aprovecharon los recursos naturales. Sin embargo, a pesar de la importancia manifiesta de este tipo de análisis, normalmente no se realizan, ya sea por desconocimiento, por falta de medios o de especialistas. En el presente trabajo se refleja este hecho para la Prehistoria Reciente de Andalucía a partir de los datos recogidos en los anuarios arqueológicos de Andalucía desde 1985 a 2004. Numéricamente la cantidad de estudios de polen, semillas, carbones, fauna o datación por carbono 14 no supera el 18\% en los 316 yacimientos arqueológicos registrados, un porcentaje realmente escaso que no ha experimentado ningún tipo de evolución (bajo un punto de vista estadístico) desde 1985.

\section{Palabras clave}

Andalucía | Anuario Arqueológico de Andalucía | Arqueozoología | Materiales arqueológicos orgánicos | Paleobiología | Prehistoria | Revisión bibliométrica | 


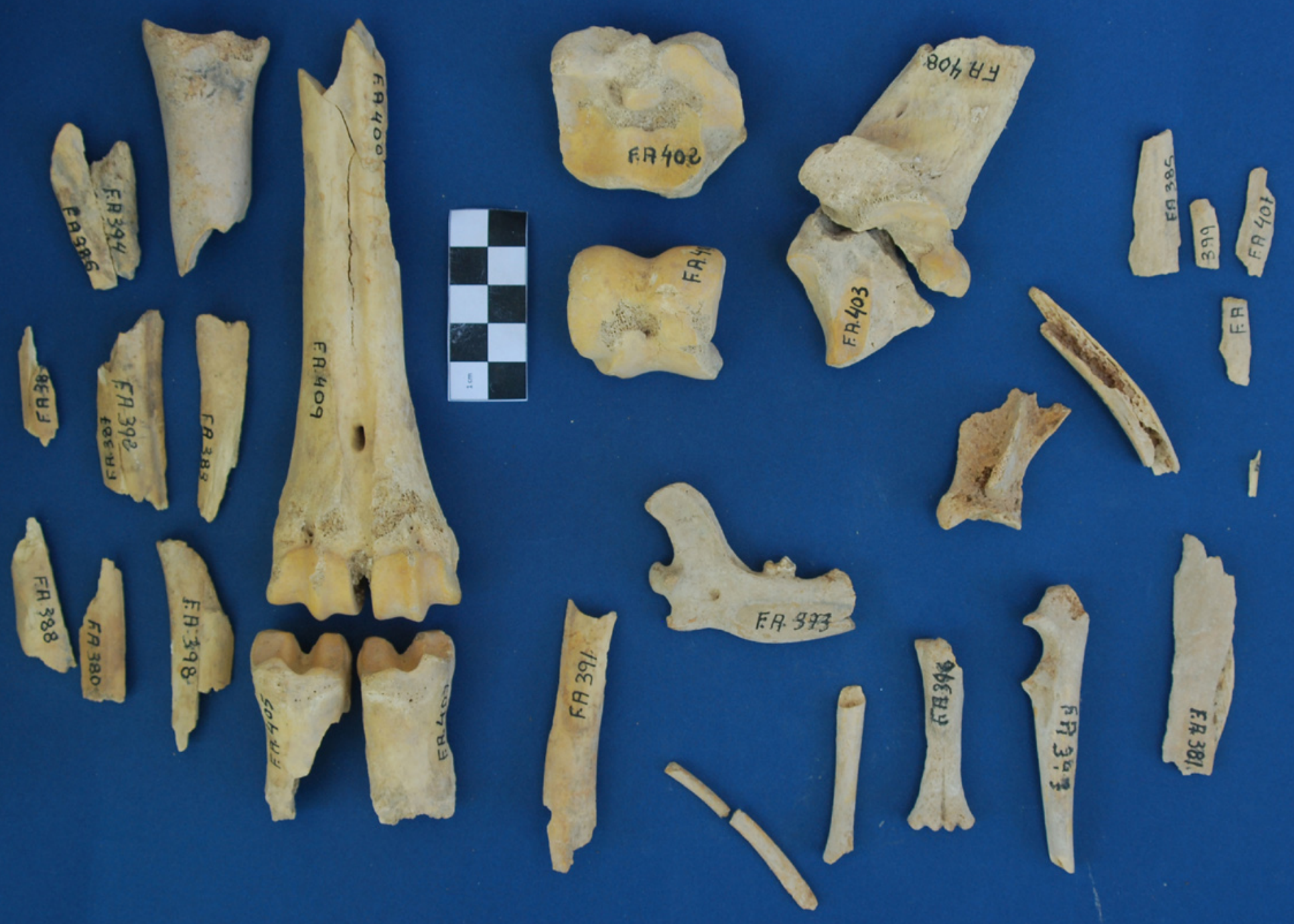

Yacimiento Cortijo Nuevo (Almería) | foto Esteban García-Viñas 
"El saber es la única propiedad que no puede perderse" Bías de Priene (filósofo griego, s. VI a. de C.)

\section{INTRODUCCIÓN}

El término paleobiología engloba el estudio de los restos animales y vegetales del pasado, así como las relaciones entre ellos y con el medio circundante. Sin embargo, para no crear confusiones, cabe destacar que nuestro equipo de investigación utiliza el término paleobiología para denominar los estudios de material faunístico procedente de yacimientos arqueológicos, mientras que en la bibliografía este mismo tipo de investigaciones se engloban bajo el término de arqueozoología o zooarqueología. No obstante, en este trabajo también se incluirán estudios polínicos, carpológicos, antracológicos, dataciones radiocarbónicas e industria ósea.

La investigación del material orgánico procedente de yacimientos arqueológicos aporta una valiosa información acerca del manejo que los humanos han hecho de los recursos naturales, de la forma en la que han interactuado con el medio y de los cambios que han producido en el mismo en su lucha por sobrevivir y reproducirse (MORENO-GARCÍA; DAVIS, 2007), lo que, al fin y al cabo, supone la meta de cualquier grupo animal. Sin embargo, dentro de la biología y la geología, la paleobiología y la paleontología son unas especialidades poco valoradas y, al mismo tiempo, en estas últimas, la investigación de yacimientos arqueológicos está muy poco considerada (BERNÁLDEZ, 1998). Pero además, dentro de la arqueología, los estudios de material orgánico aún están más infravalorados, de hecho, según datos de Bernáldez y Bernáldez (1998) en los anuarios arqueológicos de Andalucía de 1991 y 1992 sólo el 7,1\% de todos los yacimientos (no sólo de la Prehistoria Reciente) mencionaban que los estudios de fauna iban a ser realizados. Por lo tanto, y si nos permiten la expresión, nos ha tocado bailar con la más fea, pero no por ello la menos importante, ya que es la especialidad que más nos puede ayudar a comprender nuestro presente y a proyectar cambios futuros (BERNÁLDEZ, 2010).

No existen muchos estudios paleobiológicos realizados en yacimientos del territorio andaluz, pudiendo dicha escasez, para el caso concreto de los estudios de fauna, afectar a la interpretación de las actividades ganaderas en la antigüedad (ESCACENA, 2007: 259; MEDEROS, 2001). No obstante, atendiendo a la importancia de los estudios paleobiológicos para una mejor comprensión de las relaciones entre el ser humano y el medio, hemos querido comprobar empíricamente si, como cabría esperar y debido al incremento en la variedad y precisión de las técnicas analíticas, el número de estudios de material arqueológico orgánico ha ido en aumento en los últimos años. 


\section{COMPRENDER Y VALORAR NUESTRO PATRIMONIO}

Desde que el término "fósil" fuera primero utilizado por Plinio en el siglo I a. C., han sido muchas las aproximaciones que se han hecho a este vocablo. Una de las que consideramos más completas es la que plantean López y Truyols (1994) que definen los fósiles como: “...no sólo los restos materiales de antiguos organismos en un grado más o menos elevado de conservación, sino también aquellas manifestaciones de la actividad de los organismos, cuya presencia en las rocas o en otros fósiles atestigua asimismo su real existencia...".

No obstante, ¿en qué rango temporal debe datarse un resto para considerarse un fósil? ¿Son los elementos del siglo XIX fósiles? Para resolver estas cuestiones, algunos autores consideran la creación de dos grupos: todos aquellos restos con más de 13.000 años ("fósiles"), frente a todas las evidencias posteriores a esta fecha ("subfósiles") (LÓPEZ; TRUYOLS, 1994). Por lo tanto, dado que en este trabajo se considerará el registro orgánico presente en yacimientos de la Prehistoria Reciente de Andalucía, siempre se utilizará el término de subfósil.

Pero, llegados a este punto, ¿es un fósil patrimonio? ¿Y un subfósil? De serlo, ¿serían considerados como patrimonio natural o cultural?

Según la primera acepción sobre patrimonio en el Diccionario de la Real Academia de la Lengua Española, el patrimonio es una "hacienda que alguien ha heredado de sus ascendientes". Y, a su vez, por "hacienda" se entiende el "conjunto de bienes y riquezas que alguien tiene". Obviamente, éstas son unas definiciones excesivamente someras y superficiales que no nos ayudan a esclarecer si un fósil se considera o no patrimonio.

Por otra parte, se nos plantea el problema de que, tradicionalmente, el patrimonio se clasifica y define en función de sus disciplinas de estudio (arqueológico, etnográfico, histórico, científico, paleontológico, documental, archivístico...), existiendo además una separación ampliamente aceptada entre lo que se entiende por patrimonio cultural y lo que se entiende por patrimonio natural (QUEROL, 2010: 24). Aunque las únicas definiciones válidas a la hora de delimitar y proteger el patrimonio son las recogidas en la legislación (bien nacional o autonómica) ${ }^{1}$, la UNESCO nos ofrece una interpretación mucho más ajustada a nuestra ideología de trabajo: "El patrimonio de una nación lo conforman el territorio que ocupa, su flora y fauna, y todas las creaciones y expresiones de las personas que lo han habitado: sus instituciones sociales, legales y religiosas; su lenguaje y su cultura material desde las épocas históricas más antiguas. El patrimonio comprende los bienes tangibles e intangibles heredados de los antepasados; el ambiente donde se vive; los campos, ciudades y pueblos; las tradiciones y creencias que se
Véanse: Ley 16/1985 de patrimonio histórico español (BOE, n. ${ }^{\circ} 155$, de 29 de junio de 1985); Ley 14/2007, de 26 de noviembre, de patrimonio histórico de Andalucía (BOJA, n. ${ }^{\circ}$ 248 de 19 de diciembre de 2007); Ley 42/2007 del patrimonio natural y de la biodiversidad (BOE, n. ${ }^{\circ} 299$, de 14 de diciembre de 2007). 
comparten; los valores y religiosidad; la forma de ver el mundo y adaptarse a él. El patrimonio natural y cultural constituyen la fuente insustituible de inspiración y de identidad de una nación, pues es la herencia de lo que ella fue, el sustrato de lo que es y el fundamento del mañana que aspira a legar a sus hijos" (HEVIA; HIRMAS; PEÑAFIEL, 2002).

Amparados en esta definición, en la que se integra lo natural y lo cultural, vemos que es posible considerar al patrimonio en toda su amplitud y en una única dimensión, estando siempre el humano vinculado a la naturaleza y no pudiendo ser apartado de ella. Queda entonces claro que tanto fósiles como subfósiles son parte de nuestro patrimonio, y aunque las diferentes clasificaciones en torno al mismo faciliten la labor de los expertos, obviamente son los trabajos holísticos e interdisciplinares los más enriquecedores a la hora de entender las relaciones entre el humano y el medio circundante.

Partiendo de esa premisa, mostraremos en este artículo en qué medida se han llevado a cabo estudios de este tipo de patrimonio en los diferentes yacimientos de la Prehistoria Reciente de Andalucía.

\section{METODOLOGÍA}

\section{Revisión bibliométrica}

En primer lugar, se consideró llevar a cabo el inventariado de todos los yacimientos arqueológicos de la Prehistoria Reciente localizados en Andalucía con objeto de analizar si en ellos se había estudiado el material orgánico.

En este sentido, conseguir una base de datos fiel en la que se reflejara la cantidad de estudios realizados y lo suficientemente homogénea por años para realizar estudios estadísticos se convirtió en una tarea complicada (debido a la cantidad de informes no publicados o la diversidad de publicaciones que acogen este tipo de estudios).

Sopesadas las diferentes posibilidades, se decidió utilizar una única publicación de referencia, el Anuario Arqueológico de Andalucía (en adelante AAA), siguiendo un trabajo de Bernáldez y Bernáldez (1998), ya que en ella se deben informar todos los yacimientos intervenidos en una anualidad, incluyendo la descripción, los estudios realizados y los planes futuros considerados para cada yacimiento. Bien es cierto que no todos los arqueólogos publican sus hallazgos en el AAA, pero dado que esta ausencia de datos se repite en todos los tomos, podemos asumir que el error se encuentra reflejado por igual en todo nuestro estudio. 


\section{Diseño de la base de datos}

La ingente cantidad de datos recopilados debía ser debidamente estructurada para facilitar las labores de análisis estadístico. Entre las caracteríaticas generales de los asentamientos se tomaron los siguientes: nombre, localización espacial, municipio, provincia, datación y adscripción cultural. Por su parte, los datos relativos al material arqueológico orgánico fueron clasificados en cuatro categorías: presencia, ausencia, estudio realizado y estudio por realizar. Con esta clasificación se pretendía cuantificar no sólo el grado en el que este tipo de materiales se estudia, sino también en cuántos de los yacimientos este tipo de elementos eran considerados y, por lo tanto, nombrados. Para los estudios polínicos y de carbono 14 se realizó una clasificación ligeramente diferente, siendo las categorías: estudio realizado, estudio no realizado y estudio en proceso.

\section{Análisis estadístico}

El análisis estadístico planteado se basa en dos técnicas: por un lado, el estudio de tablas de contingencia con contrastes asociados; $y$, por otro, la reali-
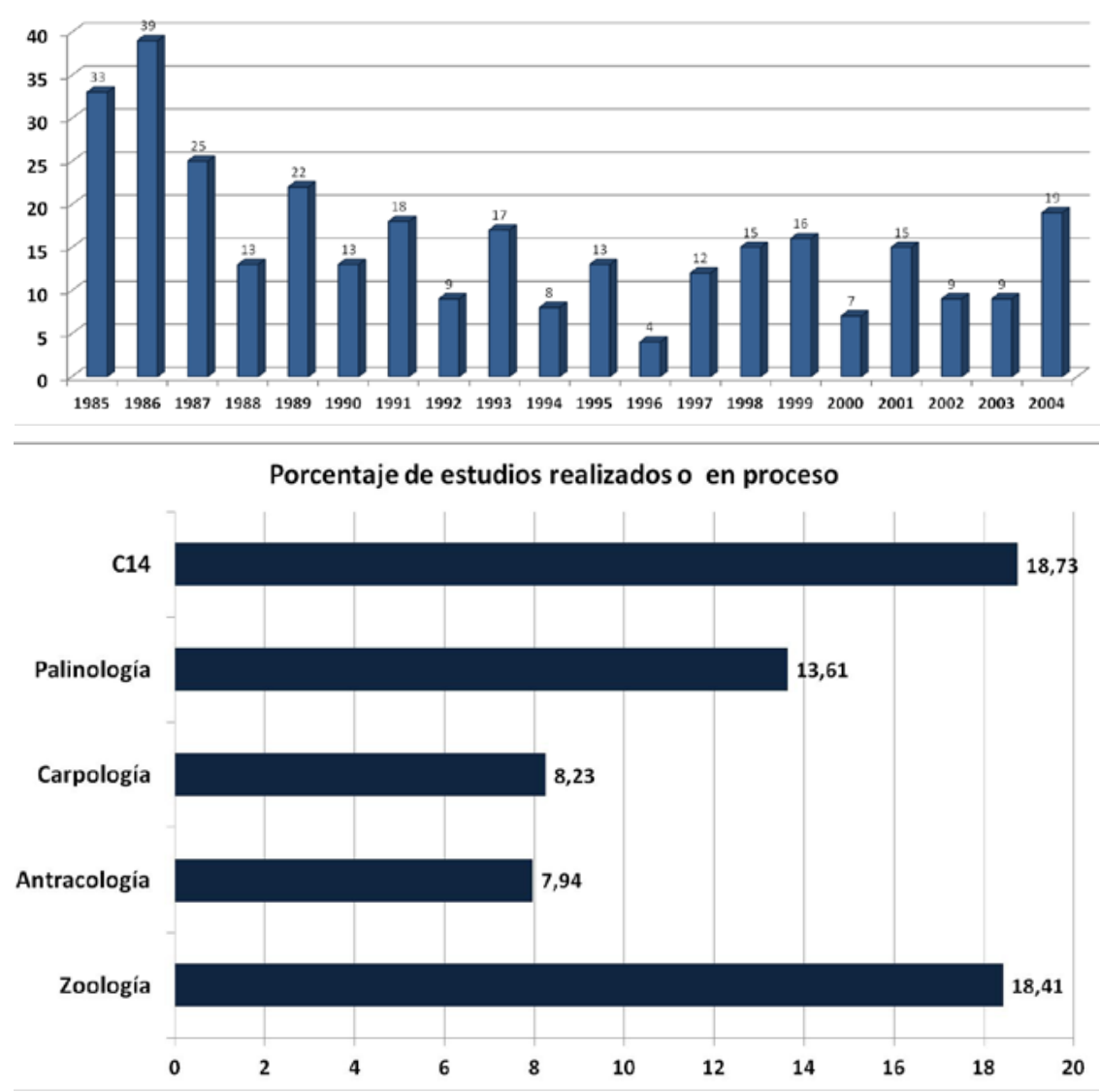

Figura 1. Representación del número de yacimientos de la Prehistoria Reciente incluidos en este estudio. Se pueden observar grandes diferencias entre las distintas anualidades, pudiendo influir éstas en la probabilidad de hallar estudios de material arqueológico orgánico | figura autores del artículo (para todos los gráficos, si no se indica lo contrario)
Figura 2. El porcentaje de estudios realizados en distintos materiales orgánicos (terminados o en proceso) no supera el $19 \%$ en ningún caso. Resultados del total de datos recopilados, 316 yacimientos | fuente GARCÍA-VIÑAS; OCAÑA; GAMERO et ál., 2013 
zación de correlaciones de Spearman de todos los parámetros en función del tiempo (MILTON, 2007). En el primero de los casos se impondrá el estadístico exacto de Fisher y la significación bilateral de Montecarlo (MILTON, 2007) ya que el número de muestras es pequeño y sólo un tipo de análisis robusto como éste puede llevarnos a obtener resultados interpretables estadísticamente. Para realizar un análisis de correlación se utilizará el porcentaje por año de cada una de las variables para reducir el error producido por la diferencia de yacimientos estudiados por cada anualidad, pues hay años en los que tenemos 39 yacimientos y en otros siete.

\section{RESULTADOS: EVOLUCIÓN DE LOS ESTUDIOS PALEOBIOLÓGICOS DURANTE 19 AÑOS}

Los resultados que se exponen a continuación están basados en la revisión bibliométrica de 24 volúmenes del Anuario Arqueológico de Andalucía, de los que se han extraído datos para 316 yacimientos (correspondientes a los periodos Calcolítico, Neolítico y Edad del Bronce). Atendiendo al gráfico reflejado en la figura 1, se observa que el número de yacimientos pertenecientes al rango temporal considerado parece haber disminuido significativamente en estos 19 años ( $p$-valor=0,025; Rho de Spearman=-0,498), aspecto que podría

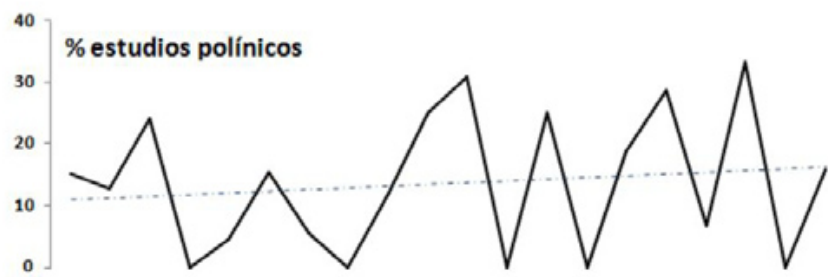

Figura 3. Evolución temporal del número de estudios de restos botánicos desde 1985 a 2004. La tendencia lineal, representada por una línea discontinua, parece ser positiva, aunque estadísticamente no se pueda apoyar esa correlación

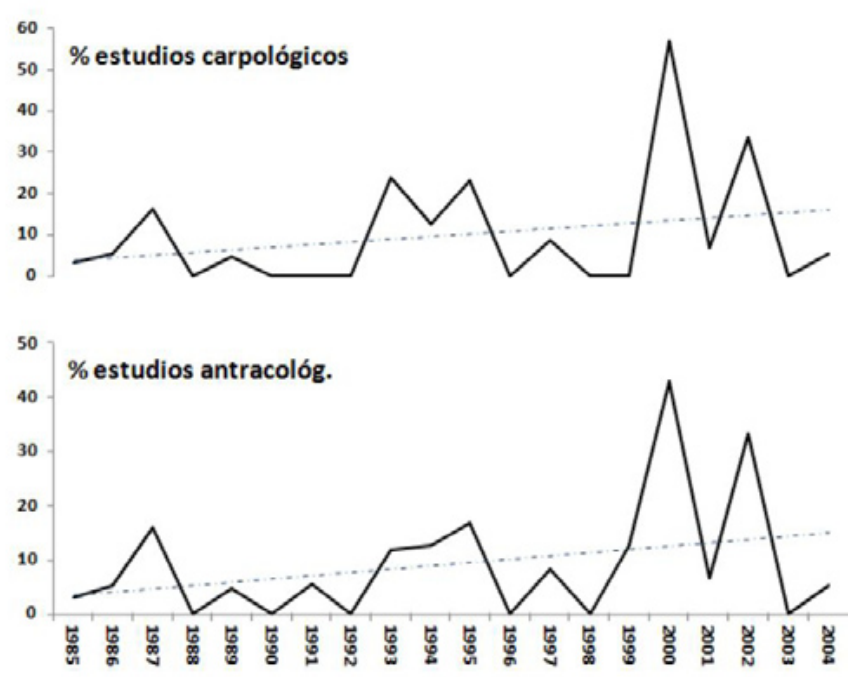


estar relacionado con el aumento de las actividades de urgencia vinculadas a la actividad constructiva en ciudades (donde a veces el registro de estructuras prehistóricas es menos abundante -o casi inexistente- en comparación con los de periodos más modernos). Con la excepción de Jaén, que cuenta con el importante yacimiento calcolítico de Marroquíes Bajos (ZAFRA; CASTRO; HORNOS, 2003), en otras capitales de provincia los yacimientos prehistóricos son escasos o están ausentes; por ejemplo, en el caso de Sevilla las estructuras más antiguas, localizadas en el yacimiento del Patio de Banderas del Real Alcázar, datan del s. IX a. C. (TABALES, 2012: 18). En nuestra base de datos este hecho aparece reflejado con sólo 30 yacimientos localizados en las capitales andaluzas, de los cuales 19 pertenecen a diferentes excavaciones dentro del área de Marroquíes Bajos (figura 1).

\section{Evolución en el estudio del material arqueológico orgánico}

En primer lugar, el resultado que más llama la atención es que, conforme a los datos totales, menos del 19\% de los 316 yacimientos incluidos en este estudio presentan análisis de material orgánico (figura 2).

En segundo lugar, según los estadísticos de Fisher y la significación de
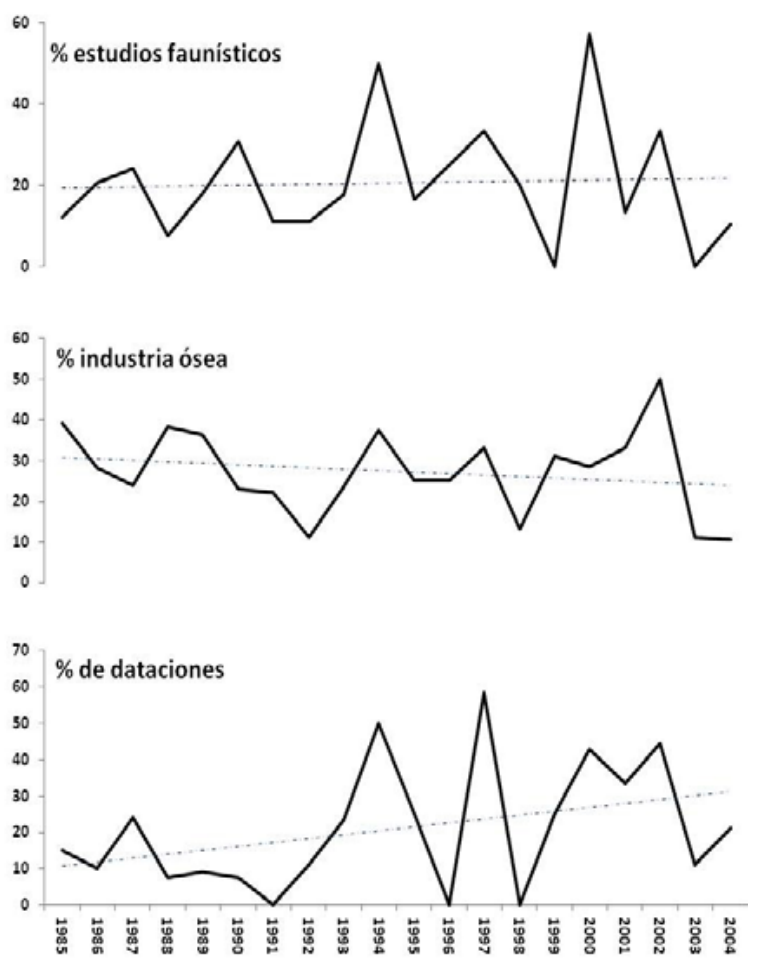

Figura 4. Evolución temporal de los estudios de fauna, industria ósea y datación C14. La tendencia lineal, representada por una línea discontinua parece mostrar ascensos o descensos (excepto para los estudios faunísticos), aunque estadísticamente no se puedan apoyar esas correlaciones 
Montecarlo, observamos que tanto los estudios de restos botánicos como las dataciones por carbono 14 muestran diferencias significativas respecto a la cantidad de análisis realizados por anualidad, no ocurriendo lo mismo en los estudios de fauna y de industria ósea (figuras 3 y 4). Es decir, hay años en los que el número total de estudios carpológicos, antracológicos, polínicos y de datación han sido superiores que en otros, pero para poder comprobar si esta diferencia se da de una manera progresiva en el tiempo se aplica un test de correlación de Spearman. Con éste se obtuvo que, de todos los análisis realizados en las excavaciones, sólo los faunísticos presentaban una correlación significativa, pero negativa ( $p$-valor $=0,036$; Rho de Spearman=-0,471), lo que quiere decir que la proporción de yacimientos con fauna estudiada había disminuido en esos 19 años.

Llegados a este punto, conviene aclarar que los resultados estadísticos anteriormente presentados son fruto del análisis de los datos totales para cada una de las categorías. Como se comentó al principio de este artículo el número de yacimientos prehistóricos incluidos en el AAA ha ido disminuyendo con los años, por lo que la probabilidad de encontrar estudios paleofaunísticos es menor en años más recientes, y esa es la tendencia que se ha detectado. No obstante, cuando relativizamos los datos en función del número total de excavaciones (esto es, porcentaje de estudios por año), ninguno de los materiales analizados presentan diferencias temporales significativas en el número de estudios (en proceso o realizados), aunque gráficamente se observe una posible tendencia positiva de los mismos, más marcada además en los resultados para la datación por C14 (figuras 2 y 3). De hecho, son los análisis por C14 los que presentan el porcentaje más alto de estudios con un $58,33 \%$ en el año 1997 (siete estudios de un total de 12 yacimientos).

Figura 5. Número de estudios de material orgánico por provincia andaluza

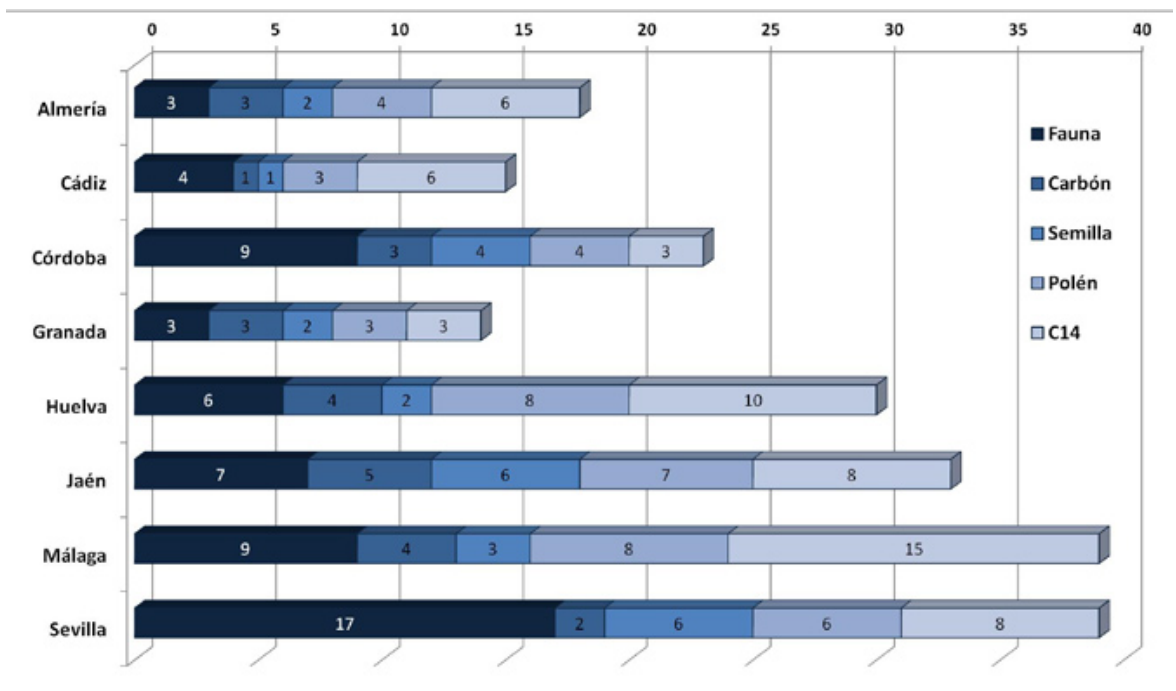




\section{Análisis de homogeneidad de estudios realizados por provincia}

La comunidad andaluza presenta un rico patrimonio arqueológico y paleontológico distribuido por todo su territorio, y con este trabajo hemos querido comprobar si los escasos estudios de material orgánico estaban concentrados geográficamente en función de las áreas de acción de los pocos especialistas que existen en estas materias.

En la figura 5 se puede observar que son las provincias de Sevilla y Málaga las que presentan un mayor número de estudios de materiales orgánicos (realizados o en proceso). Por otra parte, es Jaén la provincia que destaca en el estudio de restos macro-vegetales (semillas y carbones), y Sevilla en la que más estudios de fauna se realizan, seguidas de Málaga y Córdoba.

No obstante, según la revisión bibliométrica realizada por García-Viñas y Bernáldez (2013) son Sevilla y Granada las provincias con un mayor número de estudios de fauna publicados (figura 6). Granada destaca en cuanto a publicaciones probablemente porque, junto a Sevilla, acogen los centros de trabajo de los dos expertos con mayor recorrido en la materia. Además hay que resaltar la importante actividad investigadora que llevó a cabo el equipo de la Universidad de Múnich (Alemania) en la provincia de Granada (MORALES, 2002; RIQUELME, 2013: 142).

\section{Análisis de homogeneidad de estudios realizados por periodo histórico}

Para comprobar si los análisis de material arqueológico orgánico se realizaban preferentemente en algún periodo concreto se han agrupado los

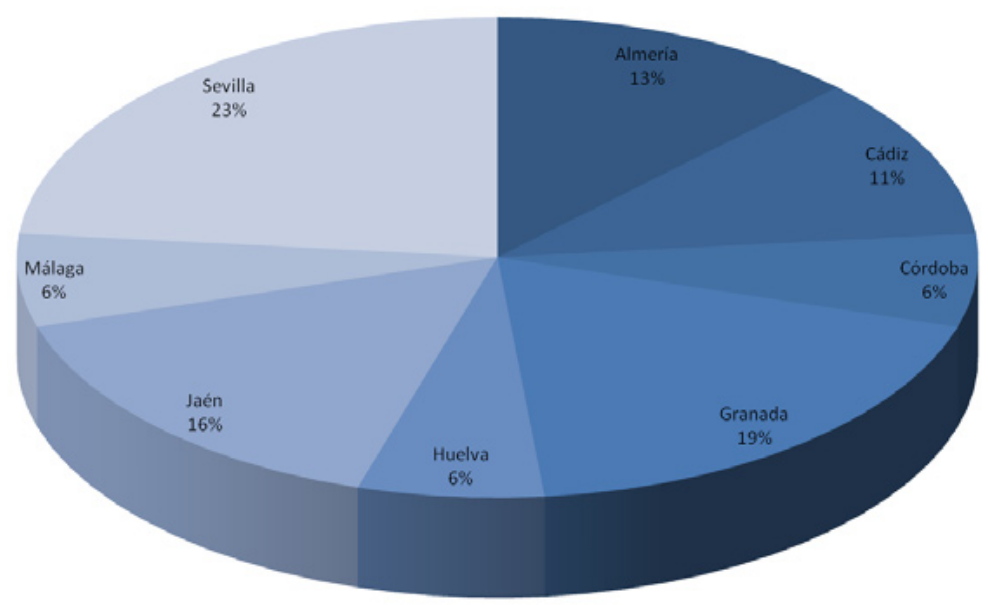

Figura 6. Porcentaje de estudios arqueozoológicos publicados por provincias andaluzas según GarcíaViñas y Bernáldez (2013). Destacan Sevilla y Granada 
Figura 7. Porcentaje de análisis de material paleobiológico (en proceso o terminados) por periodo cultural. Como se puede observar la Edad del Bronce es siempre la que menos estudios presenta según los datos extraídos de los AAA

Figura 8. En las publicaciones de arqueozoología se ha registrado un número similar de estudios para cada periodo histórico | fuente GARCÍA-VIÑAS: BERNÁLDEZ, 2013: 904
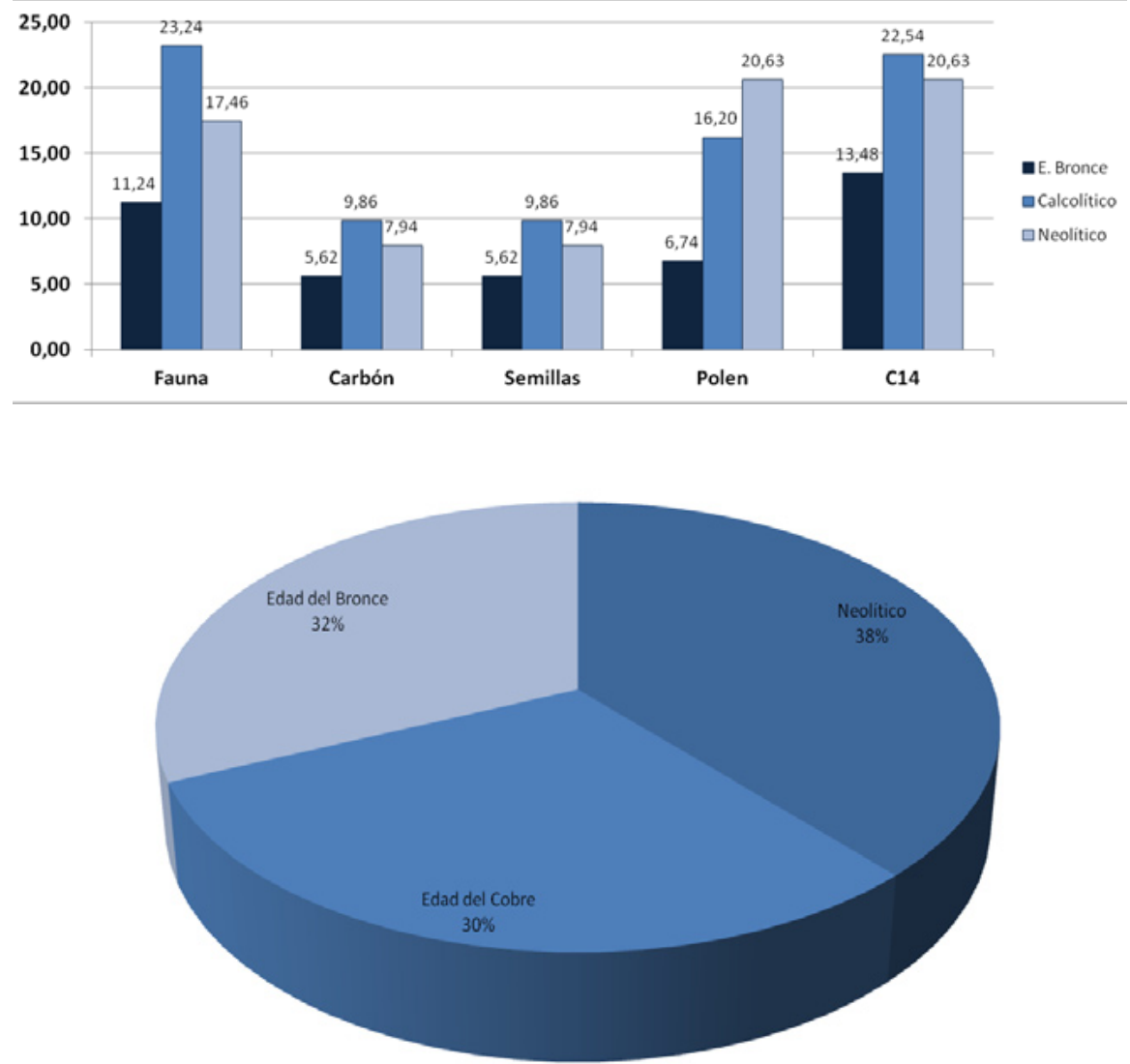

yacimientos según las dataciones aportadas por los autores de los artículos de los AAA. Pero, además, en el caso concreto de yacimientos con cronologías amplias, se han considerado de forma independiente los estratos con diferentes dataciones y se han desestimado aquéllos que no presentaban una datación suficientemente clara. Es por esto que el número de unidades (yacimientos o estratos) con el que realizamos este análisis asciende a un total de 294: 89 para la Edad del Bronce, 142 para la del Cobre y 63 para el Neolítico. Por añadidura, es importante comentar también que el número de estudios (en proceso o realizados) se ha relativizado en función del número total de los mismos por periodo para evitar la posible sobrerrepresentación del Calcolítico (ya que duplica en número de registros al Neolítico).

El resultado de la revisión de los AAA refleja en todos los casos (figura 7) que los estudios de material orgánico son más exiguos en las unidades correspondientes a la Edad del Bronce, siendo de un $11,24 \%$ en el caso concreto de los análisis de fauna, lo que aproximadamente representa la mitad de estudios respecto a los estratos del Calcolítico $(23,24 \%)$. 
No obstante, esta diferencia no parece apreciarse por García-Viñas y Bernáldez (2013) en su propio estudio bibliométrico (figura 8), lo que podría atribuirse también al trabajo del equipo de la Universidad de Múnich (Alemania), quienes publicaron antes del comienzo de los AAA, en 1985, numerosos estudios de yacimientos argáricos excavados en Granada y Almería durante los años 60, 70 y 80 del siglo XX.

\section{CONCLUSIONES}

Aunque estos resultados deben tomarse como una aproximación, nunca como datos absolutos, la primera conclusión que se puede extraer de ellos es de carácter negativo. Era lógico pensar que el número de yacimientos con estudios paleobiológicos realizados iría en aumento conforme pasara el tiempo y mejoraran las técnicas de análisis y la metodología de intervención arqueológica, no obstante, no se observan avances en la investigación del material paleobiológico en los últimos años y el material arqueológico orgánico sigue sin ser estudiado. Se podría justificar esta tendencia por el tipo de publicación con la que hemos trabajado, ya que a veces los análisis de material orgánico se realizan con mucha posterioridad al trabajo de campo y podría no quedar constancia en los AAA. No obstante, la revisión de publicaciones de estudios de fauna para este mismo periodo histórico por García-Viñas y Bernáldez (2013) refleja algo similar. Sólo se localizaron 63 publicaciones con estudios arquezoológicos en unos 40 años, siendo la primera publicación que se realizó sobre fauna de yacimientos andaluces de la Prehistoria Reciente de 1968 (BOESNECK, 1968).

Es decir, mientras que sólo un $18,4 \%$ de los 316 yacimientos registrados en el AAA presentan estudios de fauna (o al menos muestran intención de realizarlos) y sólo se han hallado 63 publicaciones de arqueozoología, en el Catálogo de patrimonio inmueble de Andalucía el número de yacimientos arqueológicos para este periodo alcanza la cifra de 3.674 (supone un 1,71\% de publicaciones arqueozoología frente a número yacimientos intervenidos -GARCÍA-VIÑAS; BERNÁLDEZ, 2013-), lo que indica que se está desaprovechando una gran cantidad de información relacionada con nuestro pasado y con la interacción entre los seres humanos y el medio.

Parte de este problema puede que sea consecuencia del tipo de arqueología practicado en los últimos años, en su mayoría intervenciones de urgencia que sólo permiten el rescate del material y la subsistencia del profesional, pero en muy pocos casos favorece una investigación profunda por falta de presupuesto y de tiempo. Quizás en este aspecto las administraciones públicas tendrían que fomentar este tipo de análisis, pues parte de lo que somos sólo se explica a través de nuestro pasado y hay que hacer todo lo posible por asegurar que ese patrimonio no se pierda. 


\section{BIBLIOGRAFÍA}

- BERNÁLDEZ, E. (2010) Basureros Arqueológicos: 8000 años de historia nos esperan. En HITA, J. M.; SUÁREZ PADILLA, J.; VILLADA PAREDES, F. (coords.) Comer en Ceuta en el siglo XIV. La alimentación durante la época mariní. Ceuta: Ciudad Autónoma de Ceuta, pp. 11-32

- BERNÁLDEZ, E. (1998) Encontré un fósil y no supe qué hacer con él. PH Boletín del IAPH, n. ${ }^{\circ} 24,1998$, pp. 64-67

- BERNÁLDEZ, E.; BERNÁLDEZ, M. (1998) El criterio paleobiológico en las ciudades históricas. En NAVAREÑO, A., MATEOS, P.; LAVADO, F. (eds.) Congreso ciudades históricas vivas. Ciudades del pasado. Mérida: Editora Regional de Extremadura, 1998, pp. 395-400

- BOESNECK, J. (1968) Restos óseos animales del Cerro de la Virgen (Orce) y del Cerro del Real (Galera), Granada. Noticiario Arqueológico Hispano, 10-11, 1968, pp. 172-189

- ESCACENA, J. L. (2007) Sobre las haciendas de Habis y de Gerión. Reflexiones para el estudio de la economía agropecuaria de Carmo en época tartésica. En BENDALA, M.; BELÉN, M. (eds.) El nacimiento de la ciudad: la Carmona protohistórica. Carmona: Universidad de Sevilla, 2007, pp. 25-73

- GARCÍA-VIÑAS, E.; BERNÁldEZ, E. (2013) Paleobiología en Andalucía: una primera aproximación a los estudios arqueozoológicos realizados para la Prehistoria reciente. En JIMÉNEZ, J.; BUSTAMANTE, M.; GARCÍA CABEZAS, M. (eds.) Actas del VI Encuentro de Arqueología del SO peninsular. Villafranca de los Barros, 4-6 de octubre de 2012. Villafranca de los Barros: Exmo. Ayuntamiento de Villafranca de los Barros, 2013, pp. 897-925

- GARCÍA-VIÑAS, E.; OCAÑA, A.; GAMERO E.; BERNÁLDEZ, E. (2012) Investigación paleobiológica en yacimientos de la Prehistoria reciente en Andalucía: estado de la cuestión. En Memorial Luis Siret. I Congreso de Prehistoria de Andalucía. La tutela del patrimonio prehistórico. Sevilla: Junta de Andalucía, 2012, pp. 615618

- HEVIA, R.; HIRMAS, C.;PEÑAFIEL, S. (2002)Patrimonio y cultura local en la escuela. Guía de experimentación e innovación pedagógica Oficina Regional de Educación, UNESCO, Santiago. Fundación Ford, 2002 [en línea] <http:// unesdoc.unesco.org/images/0015/001566/156618s.pdf> [Consulta: 14/5/2014]

- LÓPEZ, N.; TRUYOLS, J. (1994) Paleontología. Madrid: Editorial Síntesis, 1994

- MEDEROS, A. (2001) Cabaña ganadera y explotación de los productos secundarios en el sureste de la Península Ibérica (6000-1300 a. C.). En BÜCHNER, D. (ed.) Studien in memoriam wilhelm schüle. Rahden-westfalia: Verlag marie leidorf gmbh, 2001, pp. 315-331
- MILTON, J. S. (2007) Estadística para Biología y ciencias de la Salud. Madrid: McGraw Hill, 2007

- MORALES, A. (2002) 35 years of Archaeozoology in Spain: A critical review. Archaeofauna, 11, 2002, pp. 103116

- MORENO-GARCíA, M.; DAVIS, S. J. (2007) Of metapodials, measurements and music-eight years of miscellaneous zooarchaeological discoveries at the IPA, Lisbon. O Arqueólogo Português, serie IV, 25, 2007, pp. 9165

- QUEROL, M. A. (2010) Manual de gestión del patrimonio cultural. Madrid: Akal, S.A., 2010

- RIQUELME, J. A. (2013) Evolución de los estudios zooarqueológicos en Andalucía. Los últimos 25 años (19842010). En Memorial Luis Siret. I Congreso de Prehistoria de Andalucía. La tutela del patrimonio prehistórico. Sevilla: Junta de Andalucía, 2012, pp. 139-146

- TABALES, M. A. (2012) El subsuelo del Patio de Banderas entre los siglos IX a. C. y XII d. C. Campañas 2009-2012. Apuntes del Real Alcázar de Sevilla, 2012, pp. $8-53$

- ZAFRA, N.; CASTRO, F.; HORNOS, F. (2003) Sucesión y simultaneidad en un gran asentamiento: la cronología de la macro-aldea de Marroquíes Bajos, Jaén. 2500-2000 Cal ANE. Trabajos de Prehistoria, 60 (2), 2003, pp. 79-90 